\title{
Limit Definition and Pliers Mechanism
}

\author{
R. Hosseingholizadeh, A. Salimi Shamloo \\ Islamic Azad University Shabestar Branch, Iran
}

\begin{abstract}
The limit definition is the most important and the most difficult one in calculus definitions for beginners. Students always have problem in understanding it in details. In a lot of cases students cannot understand the definition or worse than that misunderstand it. This paper will try to present a teaching method for limit definition by some tools like pliers and tweezers that all students know how to work with them. Location of the notations $\forall, \exists, \epsilon$ and $\delta$ will be shown on those tools so that students have a primary sight of each part of the limit definition in her or his mind.
\end{abstract}

\section{Introduction}

Starting to learn calculus, students of science and engineering, will immediately study continuity, derivative, integral, sequences and series [1]. The meaning of limit is prerequisite all of them. Since the best definition for limit is [2]

$\exists L, \forall \epsilon>0 \exists \delta>0 \forall x 0<|x-a|<\delta \Rightarrow$ $|f(x)-L|<\epsilon \quad(1)$

Therefore understanding this logical preposition [3] is not easy. This definition is a very difficult logical preposition with various details. Each calculus book tried to describe the limit definition by mathematics methods. In some cases because of the complexity of the meaning, they start another topic without discussing it in details [4].

We know if human mind relates external experience and mathematics relations then it will be easier to understand and start using it very soon. Derivative's meaning is tied with slope of tangent line integral unite with the area under a curve. But it occurs less often that a teacher uses a pre acquainted example to his/her student's mind. Teachers usually teach limit with some or no sensible example or with just a pure description, then commence to solve different examples and to find $\delta$ from $\epsilon$ unaware of the fact that most students do not get a correct understanding from $\delta$ and $\epsilon$ that is used in the limit definition.

It will be important, if we observe that even mathematics students in some of the courses like mathematic analysis do not have any sense of the statement like $\delta \leq \frac{\varepsilon}{2} \quad$ or $\delta \leq \min \{1, \varepsilon\}$ that s/he writes. On the other hand engineering students, with this sight that the limit definition doses not have any practical interpretation, merely know calculus by derivative and integral. This paper tries to present a teaching method with which students in the first teaching class of limit get such authority that can translate meaning of limit to another in a few statements.

\section{Pliers’ mechanism}

To understand it easily, suppose we have pliers like Figure 1, handles of the pliers have been put down on $\mathrm{x}$ axis so that the handles be placed on the left and right of arbitrary point like $a$ provided that $a$ stays in the middle of the handles.

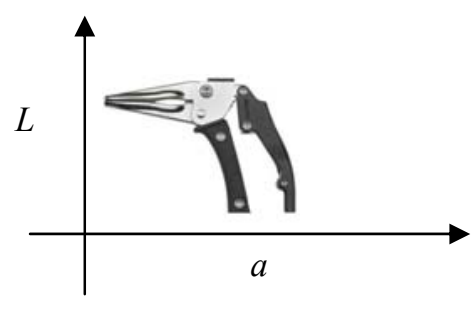

Figure 1. The pliers on axes

Let $r$ be the maximum distance between handles. If location of left handle is $\mathrm{x}_{\text {left }}$ and location of right handle is $\mathrm{x}_{\text {right }}$ then maximum distance between $\mathrm{x}_{\text {left }}$ and $x_{\text {right }}$ from a will be $r / 2$ (See Figure 2).

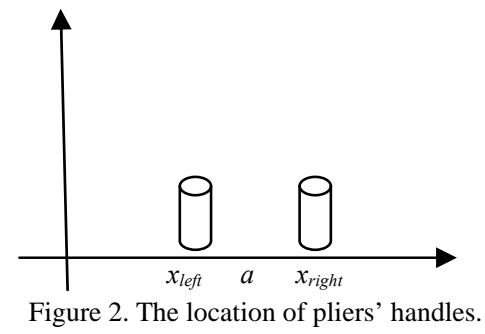

Since the pliers' jaws are in front of y axis (See Figuer1) let $r^{\prime}$ be maximum distance of jaws, let location of the bottom jaw be $y_{\text {bottom }}$ and location of the top jaw be $\mathrm{y}_{\text {top }}$ then let $\mathrm{L}$ be $\left(\mathrm{y}_{\text {top }}-\mathrm{y}_{\text {bottom }}\right) / 2$ (See Figure 3). 


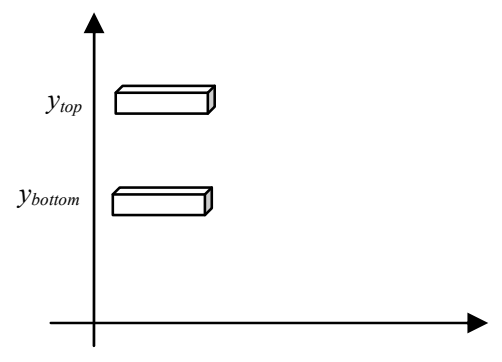

Figure 3. The location of pliers jaws.

In Figure 4 we see the pliers' mechanism that consist of two parts, opening or closing of the handles will results in opening or closing of the jaws, each of which are separately shown in Figure 2 and Figure 3 (See Figure 4).

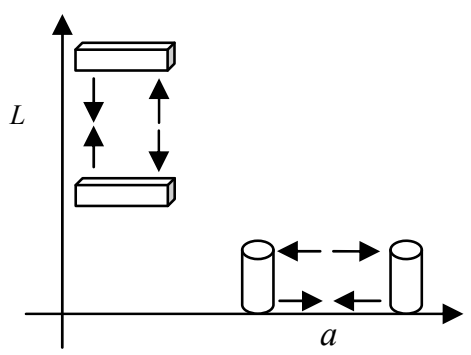

Figure 4. The location on pliers on axes

We want to show the last occurrence by mathematics notation. For this intention, distance function should be reminded first. In pre calculus distance between two points like $a, b$ on one of the axis evaluate by absolute value function that means distance between $a, b$ or $b$, $a$ is $|b-a|$. Whereas we can show all of $\mathrm{x}$ placed between $\mathrm{x}_{\text {left }}$ and $\mathrm{x}_{\text {right }}$ by $\forall x|x-a|<r$ and all of $\mathrm{y}$ placed between $\mathrm{y}_{\text {bottom }}$ and $\mathrm{y}_{\text {top }}$ by $\forall \mathrm{y}|\mathrm{y}-\mathrm{L}|<\mathrm{r}^{\prime}$.

Let there is a nail with radius $\epsilon$, hammered at the point $L$ on y axis into a wall and now we want to pull it from the wall by pliers. It will be enough to put the nail into the pliers' jaws and push the handles until the distance between top and bottom jaws is less than nail radius $\epsilon$ (See Figure 5).

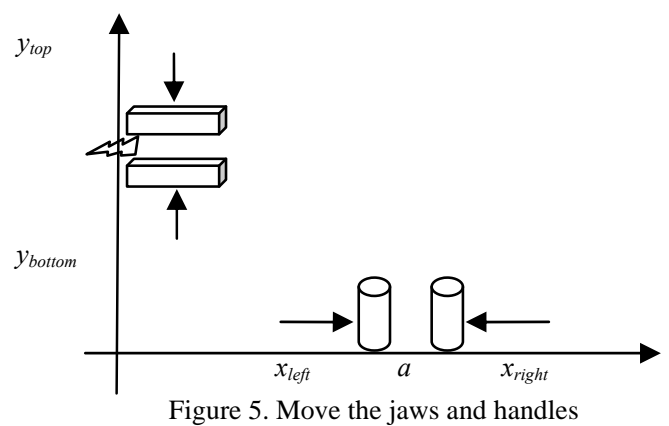

Now in this position the distance between the handles until point $a$ will be measured. We name it $\delta$.
Therefore the answer of this question, how much do the handles should be pressed to point $a$ until we can pull the nail with radius $\epsilon$ ?, is $\delta$. Our main question is that "could the pliers catch the nail if nail's radius was too small?". If someone claims his/her pliers can catch any nail with any small radius, what can I do to reject or accept his/her claim?

Obviously if you decrease the radius of the nail then you should increase the press to the handles to catch the nail between the jaws. That means for any small and smaller radius $\epsilon$ you have to find some $\delta$ to get the desirable answer. Therefore to accept this claim it will be enough for all nails with small radius $\epsilon$ to propose some $\delta$. Why dose one use a tweezers instead of pliers when she/he wants to draw out a strand of hair or thorn from her/his skin?

\section{Founding a relation between limit definition and pliers' mechanism}

The same manner should be discussed in the last section if we want to show that pliers can take every stuff with every small radius, it will be enough to show that how much the handles should be squeezed with respect to the stuff. With comparing the mechanism between pliers and tweezers in authority in picking up small stuffs we can conclude that it is possible to squeeze the pliers' handles so that the distance between handles and point of $a$ approaches zero but jaws cannot catch the stuff.

If we have ideal pliers that can hold any small stuff in its jaws, how can it be expressed in mathematics and logical style? As a matter of fact, we have to translate the following interrogative sentence:

"For any stuff with any small radius, can we squeeze the pliers' handles and decrease distance between them to decrease the distance between the jaws so that it would be smaller than the stuff to catch it between the jaws?"

Before translating some notation should be reminded:

- $\epsilon$ is the substitution arbitrary stuff radius with what we want to test the pliers' authority by (It will be constant from now on).

- $\delta$ is the distance that the handles should have from the point of $a$, to get the desirable result (if the pliers is not ideal we may not found this distance).

- If distance of $x$ from $a$ is less than $\delta$ that is $|x-a|<\delta$, then distance between the jaws will be less than $\epsilon$ that is $|y-L|<\varepsilon$ (See Figure 6). 


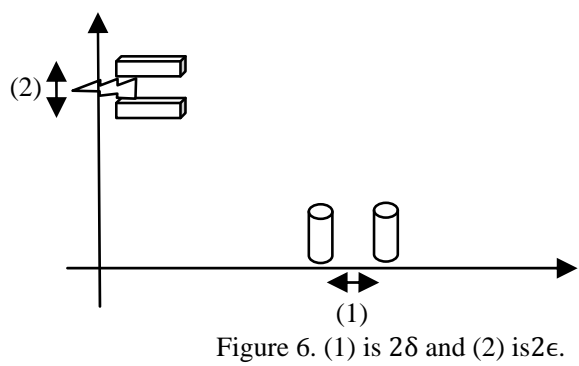

- Since in logical style the notation $\forall$ and $\exists$ respectively substitute, for all and exist then we can translate the sentence as follow:

$$
\forall \in>0 \exists \delta>0 \forall x|\mathrm{x}-\mathrm{a}|<\delta \Rightarrow|\mathrm{y}-\mathrm{L}|<
$$

$\epsilon$. (2)

Although the distance between the handles approaches zero it will be impossible until we can entirely paste the handles, therefore distance between $x$ and $a$ will not be exactly zero, then we will correct the logical sentence like this:

$\forall \epsilon>0 \exists \delta>0 \forall x 0<|\mathrm{x}-\mathrm{a}|<\delta \Rightarrow$ $|\mathrm{y}-\mathrm{L}|<\epsilon$. (3)

Since increasing or decreasing the distance between jaws depend on increasing or decreasing the distance between the handles (as a function with some carelessness) then we can let $y=f(x)$ therefore

$$
\begin{aligned}
& \forall \epsilon>0 \exists \delta>0 \forall x 0<|\mathrm{x}-\mathrm{a}|<\delta \Rightarrow \\
& |\mathrm{f}(\mathrm{x})-\mathrm{L}|<\epsilon . \text { (4) }
\end{aligned}
$$

\section{Example for nonexistence of limit}

According to our teaching experience, if the first example shows a function that is a sample of nonexistence of limit it will be impressive in understanding, and it will show the nonexistence of limit in normal position and sensible way.

\subsection{Example}

Imagine a pair of pliers, that when you squeeze its handles the distance between its jaws will open $h$ millimeters (see Figure 7).

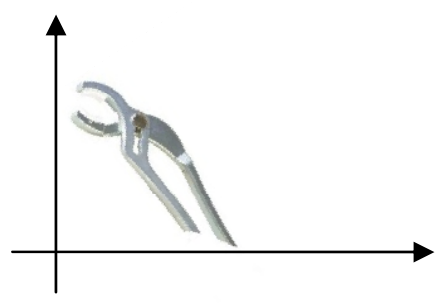

Figure 7. Nonexistence of limit

In the same way that is explained in the first section put the handles of the pliers on $\mathrm{x}$ axis so that $a$ (arbitrary point on $x$ axis) stands right in the middle of two handles, and jaws of the pliers stand in front of $y$ axis. Suppose for each millimeters decrease in distance between handles we have the same size decrease between the jaws. The function $f$ defined in (5) can show this observation.

$f(x)=\left\{\begin{array}{c}x \text { if } x<a \\ x+h \text { if } x>a\end{array}\right.$

The function figure and place of pliers' handles and jaws is illustrated in Figure 8.

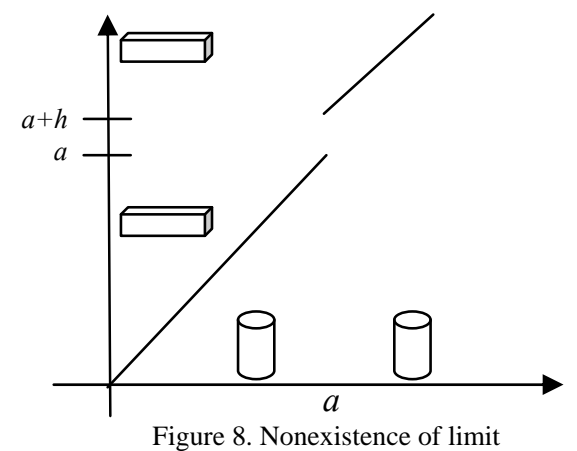

The handles approach will coincide the jaws approach as well. We press the handles so much that we cannot squeeze them anymore the same as illustrated in Figure 9, in spite of the closeness of the handles of pliers the jaws left opened $h$ millimeters that means this pliers cannot take stuff with dimension less than $h$.

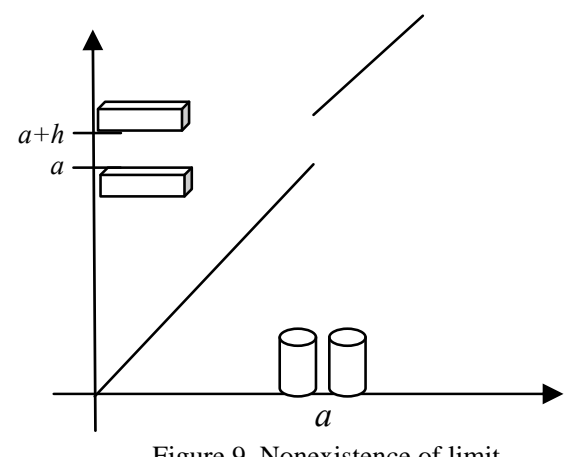

Figure 9. Nonexistence of limit

\section{Example for existence of limit}

Following is another example for existence of limit.

\subsection{Example}

Suppose that we have pliers when you press its handles two millimeters, its jaws closed one millimeter; the same as example 4.1 we put the handles of the pliers on $x$ axis so that $a$ (arbitrary point on $x$ axis) stand right in the middle of two handles, and jaws of pliers stand in front of $y$ axis. Suppose for two millimeters decrease in distance between handles we have one millimeter decrease 
between the jaws. The function $g$ defined in (6) can simulate this observation

$$
g(x)=2 x \text { if } x \neq a .(6)
$$

The function and place of pliers' handles and jaws is illustrated in Figure 10 when the handles approach each other coincide the jaws approach as well. But unlike example 4.1 if we adequately squeeze the handles we can catch any stuff with any dimension.

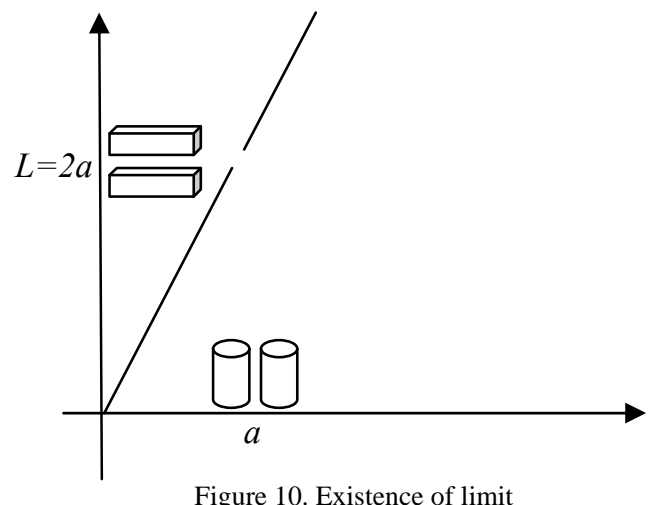

\section{Comprehension of Limit's Definition by Pliers}

Therefore when the students notice relationship between details of limit definition and pliers' mechanism, now Figure 11, 12 and 13 will be important to assemble the topics and will prepare them for the next subject, continuity. Figure 11 shows the function that has not limit at point of a .

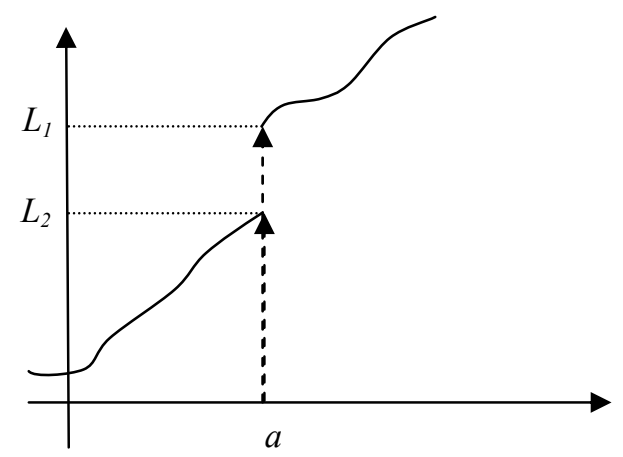

Figure 11. Nonexistence of limit

Figure 12 shows to exist limit at the point of a for shown function.

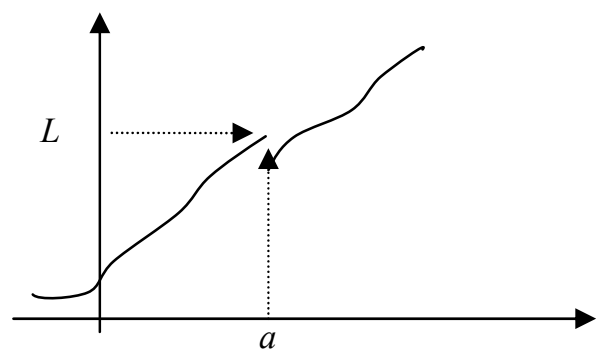

Figure 12. Existence of limit
Figure 13 shows a function that it is continues at the point of a.

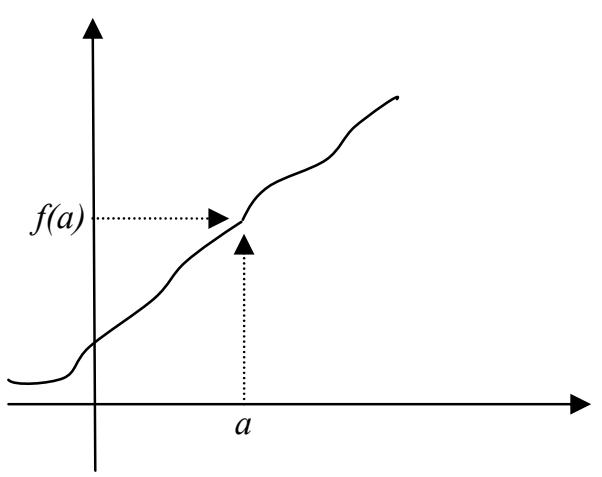

Figure 13. Continuity at the point of $a$.

Now the students remember figures 11, 12 and 13 and relationship between limit and pliers mechanism therefore they can obtain results below:

- Nonexistence at a point is not strange phenomena.

- Existence of limit at a point is necessary condition for continuity at that point.

- Continuity at a point is necessary and sufficient condition for existence of limit at that point.

Now the teacher his/her student can finish the class by three examples like below samples.

1. $y=[x]+x$ at $a=1$

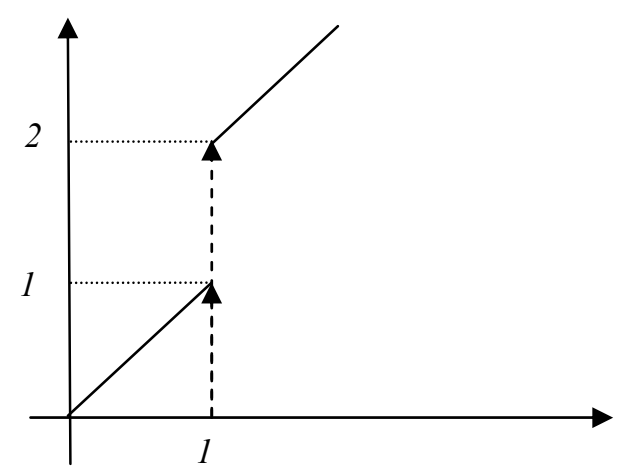

Figure 14. Nonexistence of limit

2. $y=\frac{x^{2}-1}{x-1} \quad$ at $a=1$

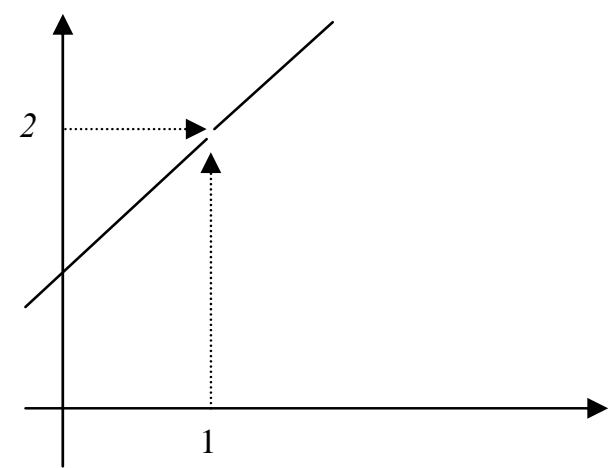

Figure 15. Existence of limit 
3. $y=x+1$ at $a=1$

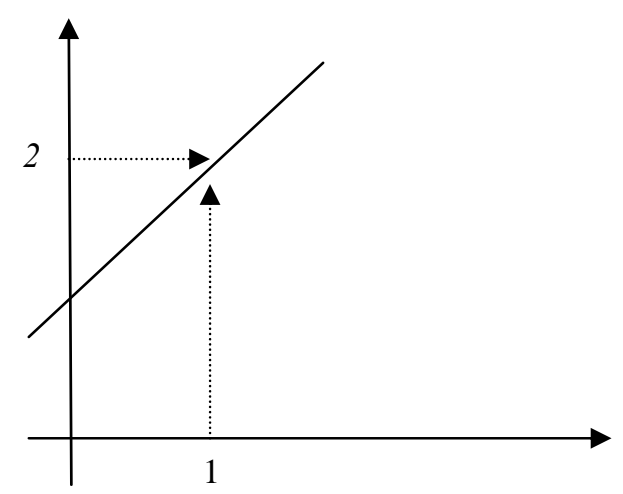

Figure 16. Continuity

\section{Conclusion}

The paper wanted to present a tangible teaching method for the limit definition, which is the gate of calculus, so that it might be rescued from its pureness and abstraction. As when a strand of a hair on a face is needed to be extracted according to its thickness, the amount of decrease between tweezers handles and closeness of the tweezers' jaws remind the details of limit definition.

Therefore the basic goal of the paper is to produce a relationship between nonexistence of limit at a point and the tweezers which cannot catch the strand of hair in its jaws.

\section{References}

[1] Tom M. Apostol, Calculus, Volume 1, second edition, Xerox Corporation, Waltham, Massachusetts, 1967.

[2] George B. Thomas, JR. Ross L. Finney, Calculus an Analytic Geometry, Published by Addison-Wesley, 1991.

[3] Gabbay, Dev M. Elementary Logics: a procedural perspective/ Doe Gabbay. Prentice Hall series in computer science, 1998.

[4] Robert T. Smith and Roland B Minton, Calculus, second edition. Published by McGraw-Hill, 2002. 